\title{
Some basic properties of certain subclasses of meromorphically starlike functions
}

\author{
Zhi-Gang Wang ${ }^{1 *}$, HM Srivastava ${ }^{2}$ and Shao-Mou Yuan ${ }^{3}$
}

"Correspondence:
zhigangwang@foxmail.com
1School of Mathematics and
Statistics, Anyang Normal University,
Anyang, Henan 455000, People's
Republic of China
Full list of author information is
available at the end of the article

available at the end of the article

\begin{abstract}
In this paper, we introduce and investigate certain subclasses of meromorphically starlike functions. Such results as coefficient inequalities, neighborhoods, partial sums, and inclusion relationships are derived. Relevant connections of the results derived here with those in earlier works are also pointed out.

MSC: Primary 30C45; secondary 30C80

Keywords: meromorphic function; starlike function; Hadamard product (or convolution); neighborhood; partial sum
\end{abstract}

\section{Introduction}

Let $\Sigma$ denote the class of functions $f$ of the form

$$
f(z)=\frac{1}{z}+\sum_{k=1}^{\infty} a_{k} z^{k}
$$

which are analytic in the punctured open unit disk

$$
\mathbb{U}^{*}:=\{z: z \in \mathbb{C} \text { and } 0<|z|<1\}=: \mathbb{U} \backslash\{0\} .
$$

A function $f \in \Sigma$ is said to be in the class $\mathcal{M S}^{*}(\alpha)$ of meromorphically starlike functions of order $\alpha$ if it satisfies the inequality

$$
\Re\left(\frac{z f^{\prime}(z)}{f(z)}\right)<-\alpha \quad(z \in \mathbb{U} ; 0 \leqq \alpha<1) .
$$

Let $\mathcal{P}$ denote the class of functions $p$ given by

$$
p(z)=1+\sum_{k=1}^{\infty} p_{k} z^{k} \quad(z \in \mathbb{U}),
$$

which are analytic in $\mathbb{U}$ and satisfy the condition

$$
\Re(p(z))>0 \quad(z \in \mathbb{U}) .
$$

For some recent investigations on analytic starlike functions, see (for example) the earlier works $[1-14]$ and the references cited in each of these earlier investigations.

O2014 Wang et al:; licensee Springer. This is an Open Access article distributed under the terms of the Creative Commons Attribution License (http://creativecommons.org/licenses/by/2.0), which permits unrestricted use, distribution, and reproduction in any medium, provided the original work is properly cited. 
Given two functions $f, g \in \Sigma$, where $f$ is given by (1.1) and $g$ is given by

$$
g(z)=\frac{1}{z}+\sum_{k=1}^{\infty} b_{k} z^{k}
$$

the Hadamard product (or convolution) $f * g$ is defined by

$$
(f * g)(z):=\frac{1}{z}+\sum_{k=1}^{\infty} a_{k} b_{k} z^{k}=:(g * f)(z) .
$$

A function $f \in \Sigma$ is said to be in the class $\mathcal{H}(\beta, \lambda)$ if it satisfies the condition

$$
\Re\left(\frac{z f^{\prime}(z)}{f(z)}+\beta \frac{z^{2} f^{\prime \prime}(z)}{f(z)}\right)<\beta \lambda\left(\lambda+\frac{1}{2}\right)+\frac{\beta}{2}-\lambda \quad(z \in \mathbb{U})
$$

where (and throughout this paper unless otherwise mentioned) the parameters $\beta$ and $\lambda$ are constrained as follows:

$$
\beta \geqq 0 \quad \text { and } \quad \frac{1}{2} \leqq \lambda<1 .
$$

Clearly, we have

$$
\mathcal{H}(0, \lambda)=\mathcal{M S}^{*}(\lambda)
$$

In a recent paper, Wang et al. [15] had proved that if $f \in \mathcal{H}(\beta, \lambda)$, then $f \in \mathcal{M S}^{*}(\lambda)$, which implies that the class $\mathcal{H}(\beta, \lambda)$ is a subclass of the class $\mathcal{M S}^{*}(\lambda)$ of meromorphically starlike functions of order $\lambda$.

Let $\mathcal{H}^{+}(\beta, \lambda)$ denote the subset of $\mathcal{H}(\beta, \lambda)$ such that all functions $f \in \mathcal{H}(\beta, \lambda)$ having the following form:

$$
f(z)=\frac{1}{z}-\sum_{k=1}^{\infty} a_{k} z^{k} \quad\left(a_{k} \geqq 0\right) .
$$

In the present paper, we aim at proving some coefficient inequalities, neighborhoods, partial sums and inclusion relationships for the function classes $\mathcal{H}(\beta, \lambda)$ and $\mathcal{H}^{+}(\beta, \lambda)$.

\section{Preliminary results}

In order to prove our main results, we need the following lemmas.

Lemma 2.1 (See [16]) If the function $p \in \mathcal{P}$ is given by (1.2), then

$$
\left|p_{k}\right| \leqq 2 \quad(k \in \mathbb{N})
$$


Lemma 2.2 Let $\beta>0$ and $1-\gamma-2 \beta>0$. Suppose also that the sequence $\left\{A_{k}\right\}_{k=1}^{\infty}$ is defined by

$$
\begin{aligned}
& A_{1}=\frac{1-\gamma-2 \beta}{1-\beta} \text { and } \\
& A_{k+1}=\frac{2(1-\gamma-2 \beta)}{1-2 \beta+(\beta k+1)(k+1)}\left(1+\sum_{l=1}^{k} A_{l}\right) \quad(k \in \mathbb{N}) .
\end{aligned}
$$

Then

$$
A_{k}=\frac{1-\gamma-2 \beta}{1-\beta} \prod_{j=1}^{k-1} \frac{3-6 \beta-2 \gamma+j(\beta j+1-\beta)}{1-2 \beta+(\beta j+1)(j+1)} \quad(k \in \mathbb{N} \backslash\{1\}) .
$$

Proof By virtue of (2.1), we easily get

$$
[1-2 \beta+(\beta k+1)(k+1)] A_{k+1}=2(1-\gamma-2 \beta)\left(1+\sum_{l=1}^{k} A_{l}\right)
$$

and

$$
[1-2 \beta+(\beta k+1-\beta) k] A_{k}=2(1-\gamma-2 \beta)\left(1+\sum_{l=1}^{k-1} A_{l}\right) .
$$

Combining (2.3) and (2.4), we obtain

$$
\frac{A_{k+1}}{A_{k}}=\frac{3-6 \beta-2 \gamma+k(\beta k+1-\beta)}{1-2 \beta+(\beta k+1)(k+1)} .
$$

Thus, for $k \geqq 2$, we deduce from (2.5) that

$$
A_{k}=\frac{A_{k}}{A_{k-1}} \cdots \frac{A_{3}}{A_{2}} \cdot \frac{A_{2}}{A_{1}} \cdot A_{1}=\frac{1-\gamma-2 \beta}{1-\beta} \prod_{j=1}^{k-1} \frac{3-6 \beta-2 \gamma+j(\beta j+1-\beta)}{1-2 \beta+(\beta j+1)(j+1)} .
$$

The proof of Lemma 2.2 is evidently completed.

The following two lemmas can be derived from [17, Theorem 1] (see also [18]), we here choose to omit the details of proof.

Lemma 2.3 Let

$$
1+\beta \lambda\left(\lambda+\frac{1}{2}\right)-\lambda-\frac{3}{2} \beta>0 .
$$

Suppose also that $f \in \Sigma$ is given by (1.1). If

$$
\sum_{k=1}^{\infty}[k+\beta k(k-1)+\gamma]\left|a_{k}\right| \leqq 1-\gamma-2 \beta,
$$


where (and throughout this paper unless otherwise mentioned) the parameter $\gamma$ is constrained as follows:

$$
\gamma:=\lambda-\beta \lambda\left(\lambda+\frac{1}{2}\right)-\frac{\beta}{2}
$$

then $f \in \mathcal{H}(\beta, \lambda)$.

Lemma 2.4 Let $f \in \Sigma$ be given by (1.5). Suppose also that $\gamma$ is defined by (2.8) and the condition (2.6) holds true. Then $f \in \mathcal{H}^{+}(\beta, \lambda)$ if and only if

$$
\sum_{k=1}^{\infty}[k+\beta k(k-1)+\gamma] a_{k} \leqq 1-\gamma-2 \beta
$$

\section{Main results}

We begin by proving the following coefficient estimates for functions belonging to the class $\mathcal{H}(\beta, \lambda)$.

Theorem 3.1 Let $\gamma$ be defined by (2.8). If $f \in \mathcal{H}(\beta, \lambda)$ with $0<\beta<2 / 5$, then

$$
\left|a_{1}\right| \leqq \frac{1-\gamma-2 \beta}{1-\beta}
$$

and

$$
\left|a_{k}\right| \leqq \frac{1-\gamma-2 \beta}{1-\beta} \prod_{j=1}^{k-1} \frac{3-6 \beta-2 \gamma+j(\beta j+1-\beta)}{1-2 \beta+(\beta j+1)(j+1)} \quad(k \in \mathbb{N} \backslash\{1\}) .
$$

Proof Suppose that

$$
q(z):=-\frac{z f^{\prime}(z)}{f(z)}-\beta \frac{z^{2} f^{\prime \prime}(z)}{f(z)}+\beta \lambda\left(\lambda+\frac{1}{2}\right)+\frac{\beta}{2}-\lambda .
$$

Then, by the definition of the function class $\mathcal{H}(\beta, \lambda)$, we know that $q$ is analytic in $\mathbb{U}$ and

$$
\Re(q(z))>0 \quad(z \in \mathbb{U})
$$

with

$$
q(0)=1-\gamma-2 \beta>0 \text {. }
$$

It follows from (2.8) and (3.1) that

$$
q(z) f(z)=-z f^{\prime}(z)-\beta z^{2} f^{\prime \prime}(z)-\gamma f(z) .
$$

By noting that

$$
h(z)=\frac{q(z)}{1-\gamma-2 \beta} \in \mathcal{P},
$$


if we put

$$
q(z)=c_{0}+\sum_{k=1}^{\infty} c_{k} z^{k} \quad\left(c_{0}=1-\gamma-2 \beta\right)
$$

by Lemma 2.1, we know that

$$
\left|c_{k}\right| \leqq 2(1-\gamma-2 \beta) \quad(k \in \mathbb{N}) .
$$

It follows from (3.2) that

$$
\begin{aligned}
& \left(c_{0}+\sum_{k=1}^{\infty} c_{k} z^{k}\right)\left(\frac{1}{z}+\sum_{k=1}^{\infty} a_{k} z^{k}\right) \\
& =\left(\frac{1}{z}-\sum_{k=1}^{\infty} k a_{k} z^{k}\right)-\left(\frac{2 \beta}{z}+\beta \sum_{k=1}^{\infty} k(k-1) a_{k} z^{k}\right)-\gamma\left(\frac{1}{z}+\sum_{k=1}^{\infty} a_{k} z^{k}\right) .
\end{aligned}
$$

In view of (3.3), we get

$$
(1-\gamma-2 \beta) a_{1}+c_{2}=-a_{1}-\gamma a_{1}
$$

and

$$
\begin{aligned}
& c_{k+2}+(1-\gamma-2 \beta) a_{k+1}+\sum_{l=1}^{k} a_{l} c_{k+1-l} \\
& =-(k+1) a_{k+1}-\beta k(k+1) a_{k+1}-\gamma a_{k+1} \quad(k \in \mathbb{N}) .
\end{aligned}
$$

From (3.4), we obtain

$$
\left|a_{1}\right| \leqq \frac{1-\gamma-2 \beta}{1-\beta}
$$

Moreover, we deduce from (3.5) that

$$
\left|a_{k+1}\right| \leqq \frac{2(1-\gamma-2 \beta)}{1-2 \beta+(\beta k+1)(k+1)}\left(1+\sum_{l=1}^{k}\left|a_{l}\right|\right) \quad(k \in \mathbb{N}) .
$$

Next, we define the sequence $\left\{A_{k}\right\}_{k=1}^{\infty}$ as follows:

$$
A_{1}=\frac{1-\gamma-2 \beta}{1-\beta} \quad \text { and } \quad A_{k+1}=\frac{2(1-\gamma-2 \beta)}{1-2 \beta+(\beta k+1)(k+1)}\left(1+\sum_{l=1}^{k} A_{l}\right) \quad(k \in \mathbb{N}) \text {. }
$$

In order to prove that

$$
\left|a_{k}\right| \leqq A_{k} \quad(k \in \mathbb{N})
$$


we make use of the principle of mathematical induction. By noting that

$$
\left|a_{1}\right| \leqq A_{1}=\frac{1-\gamma-2 \beta}{1-\beta}
$$

Therefore, assuming that

$$
\left|a_{l}\right| \leqq A_{l} \quad(l=1,2,3, \ldots, k ; k \in \mathbb{N})
$$

Combining (3.7) and (3.8), we get

$$
\begin{aligned}
\left|a_{k+1}\right| & \leqq \frac{2(1-\gamma-2 \beta)}{1-2 \beta+(\beta k+1)(k+1)}\left(1+\sum_{l=1}^{k}\left|a_{l}\right|\right) \\
& \leqq \frac{2(1-\gamma-2 \beta)}{1-2 \beta+(\beta k+1)(k+1)}\left(1+\sum_{l=1}^{k} A_{l}\right)=A_{k+1} \quad(k \in \mathbb{N}) .
\end{aligned}
$$

Hence, by the principle of mathematical induction, we have

$$
\left|a_{k}\right| \leqq A_{k} \quad(k \in \mathbb{N})
$$

as desired.

By means of Lemma 2.2 and (3.8), we know that (2.2) holds true. Combining (3.9) and (2.2), we readily get the coefficient estimates asserted by Theorem 3.1.

Following the earlier works (based upon the familiar concept of neighborhood of analytic functions) by Goodman [19] and Ruscheweyh [20], and (more recently) by Altintaş et al. [21-24], Cǎtaş [25], Cho et al. [26], Liu and Srivastava [27-29], Frasin [30], Keerthi et al. [31], Srivastava et al. [32] and Wang et al. [33]. Assuming that $\gamma$ is given by (2.8) and the condition (2.6) of Lemma 2.3 holds true, we here introduce the $\delta$-neighborhood of a function $f \in \Sigma$ of the form (1.1) by means of the following definition:

$$
\begin{aligned}
\mathcal{N}_{\delta}(f):= & \left\{g \in \Sigma: g(z)=\frac{1}{z}+\sum_{k=1}^{\infty} b_{k} z^{k}\right. \text { and } \\
& \left.\sum_{k=1}^{\infty} \frac{k+\beta k(k-1)+\gamma}{1-\gamma-2 \beta}\left|a_{k}-b_{k}\right| \leqq \delta(\delta \geqq 0)\right\} .
\end{aligned}
$$

By making use of the definition (3.10), we now derive the following result.

Theorem 3.2 Let the condition (2.6) hold true. If $f \in \Sigma$ satisfies the condition

$$
\frac{f(z)+\varepsilon z^{-1}}{1+\varepsilon} \in \mathcal{H}(\beta, \lambda) \quad(\varepsilon \in \mathbb{C} ;|\varepsilon|<\delta ; \delta>0)
$$

then

$$
\mathcal{N}_{\delta}(f) \subset \mathcal{H}(\beta, \lambda)
$$


Proof By noting that the condition (1.3) can be written as

$$
\left|\frac{\frac{z f^{\prime}(z)}{f(z)}+\beta \frac{z^{2} f^{\prime \prime}(z)}{f(z)}+1}{\frac{z f^{\prime}(z)}{f(z)}+\beta \frac{z^{2} f^{\prime \prime}(z)}{f(z)}+2 \gamma-1}\right|<1 \quad(z \in \mathbb{U})
$$

we easily find from (3.13) that a function $g \in \mathcal{H}(\beta, \lambda)$ if and only if

$$
\frac{z g^{\prime}(z)+\beta z^{2} g^{\prime \prime}(z)+g(z)}{z g^{\prime}(z)+\beta z^{2} g^{\prime \prime}(z)+(2 \gamma-1) g(z)} \neq \sigma \quad(z \in \mathbb{U} ; \sigma \in \mathbb{C} ;|\sigma|=1)
$$

which is equivalent to

$$
\frac{(g * \mathfrak{h})(z)}{z^{-1}} \neq 0 \quad(z \in \mathbb{U})
$$

where

$$
\mathfrak{h}(z)=\frac{1}{z}+\sum_{k=1}^{\infty} c_{k} z^{k} \quad\left(c_{k}:=\frac{k+\beta k(k-1)+1-[k+\beta k(k-1)+(2 \gamma-1)] \sigma}{2[\beta+(1-\gamma-\beta) \sigma]}\right) .
$$

It follows from (3.15) that

$$
\begin{aligned}
\left|c_{k}\right| & =\left|\frac{k+\beta k(k-1)+1-[k+\beta k(k-1)+(2 \gamma-1)] \sigma}{2[\beta+(1-\gamma-\beta) \sigma]}\right| \\
& \leqq \frac{k+\beta k(k-1)+1+[k+\beta k(k-1)+(2 \gamma-1)]|\sigma|}{2(1-\gamma-2 \beta)|\sigma|} \\
& =\frac{k+\beta k(k-1)+\gamma}{1-\gamma-2 \beta} \quad(|\sigma|=1) .
\end{aligned}
$$

If $f \in \Sigma$ given by (1.1) satisfies the condition (3.11), we deduce from (3.14) that

$$
\frac{(f * \mathfrak{h})(z)}{z^{-1}} \neq-\varepsilon \quad(|\varepsilon|<\delta ; \delta>0)
$$

or equivalently,

$$
\left|\frac{(f * \mathfrak{h})(z)}{z^{-1}}\right| \geqq \delta \quad(z \in \mathbb{U} ; \delta>0) .
$$

We now suppose that

$$
q(z)=\frac{1}{z}+\sum_{k=1}^{\infty} d_{k} z^{k} \in \mathcal{N}_{\delta}(f)
$$

It follows from (3.10) that

$$
\left|\frac{((q-f) * \mathfrak{h})(z)}{z^{-1}}\right|=\left|\sum_{k=1}^{\infty}\left(d_{k}-a_{k}\right) c_{k} z^{k+1}\right| \leqq|z| \sum_{k=1}^{\infty} \frac{k+\beta k(k-1)+\gamma}{1-\gamma-2 \beta}\left|d_{k}-a_{k}\right|<\delta
$$


Combining (3.16) and (3.17), we easily find that

$$
\left|\frac{(q * \mathfrak{h})(z)}{z^{-1}}\right|=\left|\frac{([f+(q-f)] * \mathfrak{h})(z)}{z^{-1}}\right| \geqq\left|\frac{(f * \mathfrak{h})(z)}{z^{-1}}\right|-\left|\frac{((q-f) * \mathfrak{h})(z)}{z^{-1}}\right|>0,
$$

which implies that

$$
\frac{(q * \mathfrak{h})(z)}{z^{-1}} \neq 0 \quad(z \in \mathbb{U}) .
$$

Therefore, we have

$$
q(z) \in \mathcal{N}_{\delta}(f) \subset \mathcal{H}(\beta, \lambda) .
$$

The proof of Theorem 3.2 is thus completed.

Next, we derive the partial sums of the class $\mathcal{H}(\beta, \lambda)$. For some recent investigations involving the partial sums in analytic function theory, one can find in $[28,29,34,35]$ and the references cited therein.

Theorem 3.3 Let $f \in \Sigma$ be given by (1.1) and define the partial sums $f_{n}(z)$ off by

$$
f_{n}(z)=\frac{1}{z}+\sum_{k=1}^{n} a_{k} z^{k} \quad(n \in \mathbb{N})
$$

If

$$
\sum_{k=1}^{\infty} \frac{k+\beta k(k-1)+\gamma}{1-\gamma-2 \beta}\left|a_{k}\right| \leqq 1
$$

where $\gamma$ is given by (2.8) and the condition (2.6) holds true, then

1. $f \in \mathcal{H}(\beta, \lambda)$;

2.

$$
\Re\left(\frac{f(z)}{f_{n}(z)}\right) \geqq \frac{n+\beta n(n+1)+2 \beta+2 \gamma}{n+\beta n(n+1)+1+\gamma} \quad(n \in \mathbb{N} ; z \in \mathbb{U}),
$$

and

$$
\Re\left(\frac{f_{n}(z)}{f(z)}\right) \geqq \frac{n+\beta n(n+1)+1+\gamma}{n+\beta n(n+1)+2-2 \beta} \quad(n \in \mathbb{N} ; z \in \mathbb{U}) .
$$

The bounds in (3.20) and (3.21) are sharp.

Proof First of all, we suppose that

$$
f_{1}(z)=\frac{1}{z} .
$$

We know that

$$
\frac{f_{1}(z)+\varepsilon z^{-1}}{1+\varepsilon}=\frac{1}{z} \in \mathcal{H}(\beta, \lambda) .
$$


From (3.19), we easily find that

$$
\sum_{k=1}^{\infty} \frac{k+\beta k(k-1)+\gamma}{1-\gamma-2 \beta}\left|a_{k}-0\right| \leqq 1
$$

which implies that $f \in \mathcal{N}_{1}\left(z^{-1}\right)$. By virtue of Theorem 3.2, we deduce that

$$
f \in \mathcal{N}_{1}\left(z^{-1}\right) \subset \mathcal{H}(\beta, \lambda)
$$

Next, it is easy to see that

$$
\frac{n+1+\beta n(n+1)+\gamma}{1-\gamma-2 \beta}>\frac{n+\beta n(n-1)+\gamma}{1-\gamma-2 \beta}>1 \quad(n \in \mathbb{N}) .
$$

Therefore, we have

$$
\sum_{k=1}^{n}\left|a_{k}\right|+\frac{n+\beta n(n+1)+1+\gamma}{1-\gamma-2 \beta} \sum_{k=n+1}^{\infty}\left|a_{k}\right| \leqq \sum_{k=1}^{\infty} \frac{k+\beta k(k-1)+\gamma}{1-\gamma-2 \beta}\left|a_{k}\right| \leqq 1
$$

We now suppose that

$$
\begin{aligned}
h_{1}(z) & =\frac{n+\beta n(n+1)+1+\gamma}{1-\gamma-2 \beta}\left(\frac{f(z)}{f_{n}(z)}-\frac{n+\beta n(n+1)+2 \beta+2 \gamma}{n+\beta n(n+1)+1+\gamma}\right) \\
& =1+\frac{\frac{n+\beta n(n+1)+1+\gamma}{1-\gamma-2 \beta} \sum_{k=n+1}^{\infty} a_{k} z^{k+1}}{1+\sum_{k=1}^{n} a_{k} z^{k+1}} .
\end{aligned}
$$

It follows from (3.22) and (3.23) that

$$
\left|\frac{h_{1}(z)-1}{h_{1}(z)+1}\right| \leqq \frac{\frac{n+\beta n(n+1)+1+\gamma}{1-\gamma-2 \beta} \sum_{k=n+1}^{\infty}\left|a_{k}\right|}{2-2 \sum_{k=1}^{n}\left|a_{k}\right|-\frac{n+\beta n(n+1)+1+\gamma}{1-\gamma-2 \beta} \sum_{k=n+1}^{\infty}\left|a_{k}\right|} \leqq 1 \quad(z \in \mathbb{U})
$$

which shows that

$$
\Re\left(h_{1}(z)\right) \geqq 0 \quad(z \in \mathbb{U}) .
$$

Combining (3.23) and (3.24), we deduce that the assertion (3.20) holds true.

Furthermore, if we put

$$
f(z)=\frac{1}{z}-\frac{1-\gamma-2 \beta}{n+\beta n(n+1)+1+\gamma} z^{n+1}
$$

then

$$
\frac{f(z)}{f_{n}(z)}=1-\frac{1-\gamma-2 \beta}{n+\beta n(n+1)+1+\gamma} z^{n+2} \rightarrow \frac{n+\beta n(n+1)+2 \beta+2 \gamma}{n+\beta n(n+1)+1+\gamma} \quad\left(z \rightarrow 1^{-}\right),
$$

which implies that the bound in (3.20) is the best possible for each $n \in \mathbb{N}$. 
Similarly, we suppose that

$$
\begin{aligned}
h_{2}(z) & =\frac{n+\beta n(n+1)+2-2 \beta}{1-\gamma-2 \beta}\left(\frac{f_{n}(z)}{f(z)}-\frac{n+\beta n(n+1)+1+\gamma}{n+\beta n(n+1)+2-2 \beta}\right) \\
& =1-\frac{\frac{n+\beta n(n+1)+2-2 \beta}{1-\gamma-2 \beta} \sum_{k=n+1}^{\infty} a_{k} z^{k+1}}{1+\sum_{k=1}^{\infty} a_{k} z^{k+1}} .
\end{aligned}
$$

In view of (3.22) and (3.26), we conclude that

$$
\left|\frac{h_{2}(z)-1}{h_{2}(z)+1}\right| \leqq \frac{\frac{n+\beta n(n+1)+2-2 \beta}{1-\gamma-2 \beta} \sum_{k=n+1}^{\infty}\left|a_{k}\right|}{2-2 \sum_{k=1}^{n}\left|a_{k}\right|-\frac{n+\beta n(n+1)+2 \beta+2 \gamma}{1-\gamma-2 \beta} \sum_{k=n+1}^{\infty}\left|a_{k}\right|} \leqq 1 \quad(z \in \mathbb{U}),
$$

which implies that

$$
\Re\left(h_{2}(z)\right) \geqq 0 \quad(z \in \mathbb{U}) .
$$

Combining (3.26) and (3.27), we readily get the assertion (3.21) of Theorem 3.3. The bound in (3.21) is sharp with the extremal function $f$ given by (3.25). We thus complete the proof of Theorem 3.3.

In what follows, we turn to quotients involving derivatives. The proof of Theorem 3.4 below is similar to that of Theorem 3.3, we here choose to omit the analogous details.

Theorem 3.4 Let $f \in \Sigma$ be given by (1.1) and define the partial sums $f_{n}(z)$ off by (3.18). If the conditions (2.6) and (3.19) hold, where $\gamma$ is given by (2.8), then

$$
\Re\left(\frac{f^{\prime}(z)}{f_{n}^{\prime}(z)}\right) \geqq \frac{(n+2) \gamma+(n+1)(n+2) \beta}{n+\beta n(n+1)+1+\gamma} \quad(n \in \mathbb{N} ; z \in \mathbb{U}),
$$

and

$$
\Re\left(\frac{f_{n}^{\prime}(z)}{f^{\prime}(z)}\right) \geqq \frac{n+\beta n(n+1)+1+\gamma}{(n-2)(n+1) \beta+2(n+1)-n \gamma} \quad(n \in \mathbb{N} ; z \in \mathbb{U}) .
$$

The bounds in (3.28) and (3.29) are sharp with the extremal function given by (3.25).

Finally, we prove the following inclusion relationship for the function class $\mathcal{H}(\beta, \lambda)$.

\section{Theorem 3.5 Let}

$$
\beta_{1} \geqq \beta_{2} \geqq 1 \quad \text { and } \quad \frac{1}{2} \leqq \lambda_{1} \leqq \lambda_{2}<1 .
$$

Then

$$
\mathcal{H}\left(\beta_{1}, \lambda_{1}\right) \subset \mathcal{H}\left(\beta_{2}, \lambda_{2}\right) .
$$

Proof Suppose that $f \in \mathcal{H}\left(\beta_{1}, \lambda_{1}\right)$. Then

$$
\Re\left(\frac{z f^{\prime}(z)}{f(z)}+\beta_{1} \frac{z^{2} f^{\prime \prime}(z)}{f(z)}\right)<\lambda_{1}\left[\beta_{1}\left(\lambda_{1}+\frac{1}{2}\right)-1\right]+\frac{\beta_{1}}{2} \quad(z \in \mathbb{U}) .
$$


Since $\beta_{1} \geqq \beta_{2} \geqq 1$ and $1 / 2 \leqq \lambda_{1} \leqq \lambda_{2}<1$, we find that

$$
\lambda_{1}\left[\beta_{1}\left(\lambda_{1}+\frac{1}{2}\right)-1\right]+\frac{\beta_{1}}{2} \leqq \lambda_{2}\left[\beta_{1}\left(\lambda_{2}+\frac{1}{2}\right)-1\right]+\frac{\beta_{1}}{2} .
$$

It follows from (3.31) and (3.32) that

$$
\Re\left(\frac{z f^{\prime}(z)}{f(z)}+\beta_{1} \frac{z^{2} f^{\prime \prime}(z)}{f(z)}\right)<\lambda_{2}\left[\beta_{1}\left(\lambda_{2}+\frac{1}{2}\right)-1\right]+\frac{\beta_{1}}{2} \quad(z \in \mathbb{U}),
$$

which shows that $f \in \mathcal{H}\left(\beta_{1}, \lambda_{2}\right)$, and subsequently, we see that $f \in \mathcal{M} S^{*}\left(\lambda_{2}\right)$, that is,

$$
\Re\left(\frac{z f^{\prime}(z)}{f(z)}\right)<-\lambda_{2} \quad(z \in \mathbb{U}) .
$$

Now, by setting

$$
\mu=\frac{\beta_{2}}{\beta_{1}}
$$

so that

$$
0<\mu \leqq 1
$$

we easily find from (3.33) and (3.34) that

$$
\begin{aligned}
& \Re\left(\frac{z f^{\prime}(z)}{f(z)}+\beta_{2} \frac{z^{2} f^{\prime \prime}(z)}{f(z)}-\lambda_{2}\left[\beta_{2}\left(\lambda_{2}+\frac{1}{2}\right)-1\right]-\frac{\beta_{2}}{2}\right) \\
& \quad=\mu \Re\left(\frac{z f^{\prime}(z)}{f(z)}+\beta_{1} \frac{z^{2} f^{\prime \prime}(z)}{f(z)}-\lambda_{2}\left[\beta_{1}\left(\lambda_{2}+\frac{1}{2}\right)-1\right]-\frac{\beta_{1}}{2}\right)+(1-\mu) \Re\left(\frac{z f^{\prime}(z)}{f(z)}+\lambda_{2}\right) \\
& \quad<0 \quad(z \in \mathbb{U}),
\end{aligned}
$$

that is,

$$
f \in \mathcal{H}\left(\beta_{2}, \lambda_{2}\right) \text {. }
$$

Therefore, the assertion (3.30) of Theorem 3.5 holds true.

From Theorem 3.5 and the definition of the function class $\mathcal{H}^{+}(\beta, \lambda)$, we easily get the following inclusion relationship.

\section{Corollary 3.6 Let}

$$
\beta_{1} \geqq \beta_{2} \geqq 1 \quad \text { and } \quad \frac{1}{2} \leqq \lambda_{1} \leqq \lambda_{2}<1 \text {. }
$$

Then

$$
\mathcal{H}^{+}\left(\beta_{1}, \lambda_{1}\right) \subset \mathcal{H}^{+}\left(\beta_{2}, \lambda_{2}\right) \subset \mathcal{M S}^{*}\left(\lambda_{2}\right) .
$$


By virtue of Lemma 2.4, we obtain the following result.

Corollary 3.7 Let $f \in \mathcal{H}^{+}(\beta, \lambda)$. Suppose also that $\gamma$ is defined by (2.8) and the condition (2.6) holds true. Then

$$
a_{k} \leqq \frac{1-\gamma-2 \beta}{k+\beta k(k-1)+\gamma}
$$

Each of these inequalities is sharp, with the extremal function given by

$$
f_{k}(z)=\frac{1}{z}-\frac{1-\gamma-2 \beta}{k+\beta k(k-1)+\gamma} z^{k} .
$$

\section{Competing interests}

The authors declare that they have no competing interests.

\section{Authors' contributions}

The authors completed the paper together. They also read and approved the final manuscript.

\section{Author details}

${ }^{1}$ School of Mathematics and Statistics, Anyang Normal University, Anyang, Henan 455000, People's Republic of China. ${ }^{2}$ Department of Mathematics and Statistics, University of Victoria, Victoria, BC V8W 3R4, Canada. ${ }^{3}$ School of Mathematics and Computing Science, Changsha University of Science and Technology, Changsha, Hunan 410114, People's Republic of China.

\section{Acknowledgements}

The present investigation was supported by the National Natural Science Foundation under Grant nos. 11301008 11226088, 11301041 and 11101053, the Foundation for Excellent Youth Teachers of Colleges and Universities of Henan Province under Grant no. 2013GGJS-146, and the Natural Science Foundation of Educational Committee of Henan Province under Grant no. 14B110012 of the People's Republic of China. The authors would like to thank the referees for their valuable comments and suggestions, which essentially improved the quality of this paper.

Received: 9 October 2013 Accepted: 5 January 2014 Published: 24 Jan 2014

\section{References}

1. Ali, RM, Ravichandran, V: Classes of meromorphic $\alpha$-convex functions. Taiwan. J. Math. 14, 1479-1490 (2010)

2. Ali, RM, Ravichandran, $\mathrm{V}$, Seenivasagan, N: Subordination and superordination of the Liu-Srivastava linear operator on meromorphic functions. Bull. Malays. Math. Soc. 31, 193-207 (2008)

3. Ali, RM, Ravichandran, V, Seenivasagan, N: On subordination and superordination of the multiplier transformation for meromorphic functions. Bull. Malays. Math. Soc. 33, 311-324 (2010)

4. Liu, J-L, Srivastava, HM: Some convolution conditions for starlikeness and convexity of meromorphically multivalent functions. Appl. Math. Lett. 16, 13-16 (2003)

5. Liu, M-S, Zhu, Y-C, Srivastava, HM: Properties and characteristics of certain subclasses of starlike functions of order $\beta$. Math. Comput. Model. 48, 402-419 (2008)

6. Mohd, MH, Ali, RM, Keong, LS, Ravichandran, V: Subclasses of meromorphic functions associated with convolution. J. Inequal. Appl. 2009, Article ID 190291 (2009)

7. Nunokawa, M, Ahuja, OP: On meromorphic starlike and convex functions. Indian J. Pure Appl. Math. 32, 1027-1032 (2001)

8. Silverman, H, Suchithra, K, Stephen, BA, Gangadharan, A: Coefficient bounds for certain classes of meromorphic functions. J. Inequal. Appl. 2008, Article ID 931981 (2008)

9. Srivastava, HM, Khairnar, SM, More, M: Inclusion properties of a subclass of analytic functions defined by an integral operator involving the Gauss hypergeometric function. Appl. Math. Comput. 218, 3810-3821 (2011)

10. Srivastava, HM, Yang, D-G, Xu, N: Some subclasses of meromorphically multivalent functions associated with a linear operator. Appl. Math. Comput. 195, 11-23 (2008)

11. Sun, Y, Kuang, W-P, Liu, Z-H: Subordination and superordination results for the family of Jung-Kim-Srivastava integral operators. Filomat 24, 69-85 (2010)

12. Tang, H, Deng, G-T, Li, S-H: On a certain new subclass of meromorphic close-to-convex functions. J. Inequal. Appl. 2013, $164(2013)$

13. Wang, Z-G, Sun, $Y, X u, N$ : Some properties of certain meromorphic close-to-convex functions. Appl. Math. Lett. 25 454-460 (2012)

14. Yuan, S-M, Liu, Z-M, Srivastava, HM: Some inclusion relationships and integral-preserving properties of certain subclasses of meromorphic functions associated with a family of integral operators. J. Math. Anal. Appl. 337, 505-515 (2008)

15. Wang, Z-G, Liu, Z-H, Xiang, R-G: Some criteria for meromorphic multivalent starlike functions. Appl. Math. Comput. 218, 1107-1111 (2011) 
16. Goodman, AW: Univalent Functions, vol. 1. Polygonal Publishing House, Washington, New Jersey (1983)

17. Dziok, J: Classes of meromorphic functions associated with conic regions. Acta Math. Sci. 32, 765-774 (2012)

18. Dziok, J: Classes of multivalent analytic and meromorphic functions with two fixed points. Fixed Point Theory Appl. 2013, 86 (2013)

19. Goodman, AW: Univalent functions and nonanalytic curves. Proc. Am. Math. Soc. 8, 598-601 (1957)

20. Ruscheweyh, S: Neighborhoods of univalent functions. Proc. Am. Math. Soc. 81, 521-527 (1981)

21. Altintaş, O: Neighborhoods of certain $p$-valently analytic functions with negative coefficients. Appl. Math. Comput. $187,47-53$ (2007)

22. Altintaş, O, Owa, S: Neighborhoods of certain analytic functions with negative coefficients. Int. J. Math. Math. Sci. 19 797-800 (1996)

23. Altintaş, O, Özkan, Ö, Srivastava, HM: Neighborhoods of a class of analytic functions with negative coefficients. Appl. Math. Lett. 13(3), 63-67 (2000)

24. Altintaş, O, Özkan, Ö, Srivastava, HM: Neighborhoods of a certain family of multivalent functions with negative coefficients. Comput. Math. Appl. 47, 1667-1672 (2004)

25. Cǎtaş, A: Neighborhoods of a certain class of analytic functions with negative coefficients. Banach J. Math. Anal. 3 , 111-121 (2009)

26. Cho, NE, Kwon, OS, Srivastava, HM: Inclusion relationships for certain subclasses of meromorphic functions associated with a family of multiplier transformations. Integral Transforms Spec. Funct. 16, 647-658 (2005)

27. Liu, J-L, Srivastava, HM: A linear operator and associated families of meromorphically multivalent functions. J. Math. Anal. Appl. 259, 566-581 (2001)

28. Liu, J-L, Srivastava, HM: Classes of meromorphically multivalent functions associated with the generalized hypergeometric function. Math. Comput. Model. 39, 21-34 (2004)

29. Liu, J-L, Srivastava, HM: Subclasses of meromorphically multivalent functions associated with a certain linear operator. Math. Comput. Model. 39, 35-44 (2004)

30. Frasin, BA: Neighborhoods of certain multivalent functions with negative coefficients. Appl. Math. Comput. 193, 1-6 (2007)

31. Keerthi, BS, Gangadharan, A, Srivastava, HM: Neighborhoods of certain subclasses of analytic functions of complex order with negative coefficients. Math. Comput. Model. 47, 271-277 (2008)

32. Srivastava, HM, Eker, SS, Seker, B: Inclusion and neighborhood properties for certain classes of multivalently analytic functions of complex order associated with the convolution structure. Appl. Math. Comput. 212, 66-71 (2009)

33. Wang, Z-G, Yuan, X-S, Shi, L: Neighborhoods and partial sums of certain subclass of starlike functions. J. Inequal. Appl. 2013, $163(2013)$

34. Aouf, MK, Mostafa, AO: On partial sums of certain meromorphic $p$-valent functions. Math. Comput. Model. 50 1325-1331 (2009)

35. Frasin, BA: Generalization of partial sums of certain analytic and univalent functions. Appl. Math. Lett. 21, 735-741 (2008)

\section{Submit your manuscript to a SpringerOpen ${ }^{\circ}$ journal and benefit from:}

- Convenient online submission

Rigorous peer review

- Immediate publication on acceptance

- Open access: articles freely available online

- High visibility within the field

- Retaining the copyright to your article 